\title{
INTRODUCTION TO THE SPECIAL ISSUE ON COLLECTIVE ADAPTIVE SYSTEMS
}

Collective Adaptive Systems (CAS) is a broad term that describes large scale systems that comprise of many units/nodes, each of which may have their own individual properties, objectives and actions. Decision-making in such a system is distributed and possibly highly dispersed, and interaction between the units may lead to the emergence of unexpected phenomena. CASs are open, in that nodes may enter or leave the collective at any time, and boundaries between CASs are fluid. The units can be highly heterogeneous (computers, robots, agents, devices, biological entities, etc.), each operating at different temporal and spatial scales, and having different (potentially conflicting) objectives and goals, even if often the system has a global goal that is pursued by means of collective actions. Our society increasingly depends on such systems, in which collections of heterogeneous technological nodes are tightly entangled with human and social structures to form artificial societies. Yet, to properly exploit them, we need to develop a deeper scientific understanding of the principles by which they operate, in order to better design them.

The aim of this special issue is to provide a selection of the state of the art, emerging trends, new technologies and best practices in the field of collective adaptive systems. The idea was born at the second FoCAS Workshop on Fundamentals of Collective Adaptive Systems at SASO 2014 in London, however, an open call enabled any researcher working on a related topic to submit a paper for review.

This special issue features four articles that concern collective adaptive systems.

The first one, "On Intention-Propagation-Based Prediction in Autonomously Self-adapting Navigation", by László Z. Varga considers road-traffic systems as an example of a collective adaptive system, and applies intention-propagation-based approaches to forecast travel times. The authors find that using intentionpropagation-based traffic forecast provides an important improvement to a simple naive online strategy, and also point out some surprising results; for example, if vehicles use a simple naive strategy and exploit intentionpropagation-based prediction, then in some networks and in some cases the traffic may be worse off by exploiting real-time information than without exploiting real-time information. They also suggest other collective adaptive systems to which the techniques could be applied, for instance manufacturing and cloud computing.

The second one, "Application of collective movement in real life scenarios: overview of current flocking solutions", by Bernát Wiandt, András Kökuti, Vilmos Simon considers collective movement of dynamic nodes within an adaptive system, addressing the issue of how to control the nodes in such a way that they behave as a coherent group (flock). The authors critically survey the existing flocking algorithms, showing that the majority are unsuitable for real world use due to overlooking crucial physical limitations or lack of sufficiently detailed models. The paper provides clear directions for future work based on a comprehensive survey and analysis of real-world scenarios.

The third paper, "Overlay Service Computing - Modular and Reconfigurable Collective Adaptive Systems", by Evangelos Pournaras, addresses the design of overlay layers in network services that support collective adaptive systems. The author introduces the notion of overlay services that provide generic application capabilities of a broad application scope enabled by one or more overlay networks. In the paper the ASMA architecture is presented, which supports the definition of structures of overlay services and guides their development by means of a simple high-level notation. The paper reports also two examples of overlay services developed according to ASMA.

The fourth paper, "SODAP: Self-Organized Topology Protection For Superpeer P2P Networks", by Paul L. Snyder and Giuseppe Valetto, addresses peer-to-peer systems organized in superpeer overlays. To address the vulnerability problems of the superpeers, the authors propose SODAP (Self-Organized Degree Adaptation Protection), which is a self-organized strategy for the self-protection of the overlay. The proposed approach is based on the local adaptation of a peer's degree in response to disconnections, by creating redundant connections that lead to a more resilient topology of the system. The authors provide an evaluation of the proposed approach by means of Myconet, a self-organized superpeer overlay for unstructured peer-to-peer networks.

We would like to thank the editorial board of SCPE for the chance of arranging this special issue, and all the reviewers for their hard work.

Giacomo Cabri and Emma Hart 